\title{
Selecting noise source and traffic representations that capture road traffic noise dynamics near traffic signals
}

Arnaud Can, Laboratoire d'Ingénierie Circulation Transports (LICIT), ENTPE / INRETS ; Université de Lyon, France. Tel : +334720477 15. Fax: +334720477 12.e-mail: can@entpe.fr.

Ludovic Leclercq, Laboratoire d'Ingénierie Circulation Transports (LICIT), ENTPE / INRETS ; Université de Lyon, France.

Joël Lelong, Laboratoire Transport Environnement (LTE), INRETS ; France. 


\section{ABSTRACT}

Considering traffic dynamics greatly improves noise estimation in urban area. This can be achieved by coupling a dynamic traffic model with both noise emission laws and sound propagation calculation. Determining the relevant noise source and traffic representations to estimate classical noise descriptors ( $L_{\mathrm{Aeq}}$ and statistical descriptors) near traffic signals has been recently studied. This research topic is extended in this paper to more specific descriptors which are able to capture noise dynamics at the traffic signal scale, for usual urban traffic situations (upstream, in front of, and downstream a traffic signal) and different distances from the road (5.5, 10 and $15 \mathrm{~m}$ ). It appears that $14 \mathrm{~m}$-line sources ensure an estimation of all descriptors with errors below $2 \mathrm{~dB}(\mathrm{~A})$ if traffic dynamics is precisely described. Macroscopic and microscopic car-following models are both relevant to highlight noise dynamics triggered by the traffic signal, but some differences between those traffic representations are observed.

\section{INTRODUCTION}

Traffic noise prediction models should consider traffic dynamics to precisely assess noise variations in urban area [1][2]. This can be done by coupling a dynamic traffic model with both emission laws and sound propagation calculation [3-8].

\begin{tabular}{|c|c|c|c|c|c|c|}
\hline Dynamic & $\begin{array}{c}v(t), x(t) \\
a(t)\end{array}$ & $\begin{array}{l}\text { Dynamic } \\
\text { Dyccion }\end{array}$ & $L_{w}(t)$ & Sound & $\begin{array}{l}L_{\text {Aeq, 1s }}(t) \\
\text { evolution }\end{array}$ & Noise \\
\hline Modelling & $\begin{array}{c}\text { for each } \\
\text { vehicle class }\end{array}$ & Calculation & $\begin{array}{l}\text { for each } \\
\text { vehicle }\end{array}$ & Calculation & & Caluclation \\
\hline
\end{tabular}

Figure 1: Modeling chain of dynamic noise estimation models

The traffic model gives position $x(t)$, speed $v(t)$ and acceleration $a(t)$ of each vehicle on the network at time $t$. Those variables are updated at each simulation time step (usually chosen between $0.5 \mathrm{~s}$ to $1 \mathrm{~s})$. They are input into emission laws to calculate noise emissions $L_{w}(t)$. Then a propagation calculation provides A-weighted equivalent sound pressure levels $L_{\text {Aeq,1s }}(t)$ for a grid of receivers. Classical descriptors but also refined descriptors that are able to capture noise dynamics at the traffic signal scale can finally be derived [9][10] (see Figure 1). Thereby, this modelling chain permits to account for traffic noise dynamics when evaluating urban traffic management policies. This offers a substantial breakthrough in traffic noise prediction since it allows for evaluating not only noise levels but also noise variations. This has been shown to be important when assessing urban noise quality [11][12]. 
Confidence bounds for the errors generated by each block will help to characterize the accuracy of the whole modelling chain. Relevant traffic and noise source representations to estimate classical descriptors ( $L_{\mathrm{Aeq}}$ and statistical descriptors) estimation under urban conditions have been determined in [13]. It has been demonstrated that: (i) individualized representation of vehicles with the same averaged driving behaviour is sufficient for classical descriptors estimation, (ii) gathering vehicle emissions on line sources is necessary to reduce calculation times; it does not affect the overall estimation provided that traffic dynamics is precisely described, (iii) line sources can spread up to $56 \mathrm{~m}$ for $L_{\text {Aeq }}$ estimation and up to $28 \mathrm{~m}$ for statistical descriptors estimation. However, this study only considered receivers quite far from the road (15m) and only focused on classical descriptors from legislation.

The contribution of the paper is to extend this study to more specific descriptors that reveal noise dynamics at the signal scale (rather than at a more aggregated scale). To specify which traffic and noise source representations are the most relevant for those descriptors estimation, we chose several receivers located upstream, in front of, and downstream a traffic signal. Three distances between the receivers and the road axis are considered to account for the increase in dynamics closer to the road: $5.5 \mathrm{~m}, 10 \mathrm{~m}$ and $15 \mathrm{~m}$. Finally, both saturated and unsaturated traffic conditions are tested by assuming different average flow rates. For comparison, the same emission law is supposed for each vehicle [14], and only geometric attenuation is considered when calculating noise propagation.

Background materials on traffic modeling, noise source representation and specific descriptors are first provided. Noise source and traffic representations are then tested separately. Actually, the paper concludes with the modeling conditions that guarantee a correct estimation of noise dynamics in urban area.

\section{BACKGROUND}

\subsection{Traffic modeling}

Traffic models are used to predict the time evolution of the key traffic variables (densities, vehicles speed and acceleration...) along the network. Traffic can be described either microscopically or macroscopically to achieve this prediction [15]. The three traffic representations tested in [13] are kept in this study: a macroscopic conservation law model (MCL model), a macroscopic car-following model (MCF model) and a microscopic car-following model (mCF model). More details on the traffic representations can be found in [13].

\subsubsection{Macroscopic Conservation law model (MCL model)}


In macroscopic models, interactions between vehicles are globally studied. Traffic is considered as a homogenous and continuous stream, by analogy to fluid dynamics. Under urban conditions, the network can be described by three parameters: the maximal speed $u$ reached when traffic is free, the wave speed $w$ at which a congestion spills back on the network, and the maximal density $\kappa$ reached when all vehicles are stopped in a queue. Each link of the network is discretized into cells whose length is $\Delta x$ ( $\Delta x=7 \mathrm{~m}$ in this study). The cumulative number of vehicles $N(x, t)$ that have crossed a point at a distance $x$ from the origin by time $t$ is updated from (1) [16][17]:

$$
N(x, t)=\min (\underbrace{N(x-\Delta x, t-\Delta t)}_{\text {demand term }}, \underbrace{N(x+\Delta x, t-u \Delta t / w)+\kappa \Delta x}_{\text {supply term }})
$$

$N$ equals the demand term when traffic is free, and equals the supply term when traffic is congested. The flow $q=\partial_{t} N$, the density $k=-\partial_{x} N$ and the speed $V=q / k$ can be deduced afterwards for each cell of the network at each time step.

\subsubsection{Macroscopic car-following model (MCF model)}

MCF is the vehicle representation of MCL models [18][19]. It has therefore the same parameters The vehicle parameters are $u, w$ and the minimum spacing $s_{\min }$ between two vehicles. This spacing is observed when vehicles are stopped for example at a traffic signal, thus $S_{\min }=1 / \kappa$. Position of vehicle $i$ at the next time step $x_{i}(t+\Delta t)$ is the minimum between the position it is willing to reach when traffic is free and the position it cannot overpass due to the downstream vehicle $i-1$ when traffic is congested:

$$
x_{i}(t+\Delta t)=\min (\underbrace{x_{i}(t)+u \Delta t}_{\text {position when traffic is free }}, \underbrace{x_{i-1}(t)-s_{\text {min }}}_{\text {position when traffic is congested }})
$$

Speed $v_{\mathrm{i}}(t)$ and acceleration $a_{\mathrm{i}}(t)$ can then be deduced from positions $x_{\mathrm{i}}(t)$ and $x_{\mathrm{i}}(t+\Delta t)$.

Note that MCF model can account for stochastic traffic flows by using shifted exponential distributions to represent vehicle arrivals. In this case, several simulation runs should be conducted for the same traffic scenario in order to cover the whole range of the possible traffic evolutions.

\subsubsection{Microscopic car-following model (mCF model)}

MCF and mCF models have both a vehicle representation, but they differ in the rule that governs driving behaviors. In MCF models it is an average rule for all vehicles while in mCF models it differs from one vehicle to the others. Each vehicle adapts its speed with respect to its leader 
(the vehicle immediately downstream), based on its own characteristics (desired spacing, speed and acceleration...) [20][21]. Outputs of the mCF model are $x_{\mathrm{i}}(t), v_{\mathrm{i}}(t)$ and $a_{\mathrm{i}}(t)$. The mCF model used in this study is described in [22]. It is representative of the car-following models classically used within traffic microsimulation tools.

Individualization of behavior rules with $\mathrm{mCF}$ allows for catching both heterogeneity and stochastic effects in traffic flow, which could improve noise estimation. Particularly, the mCF model explicitly reproduces variations from one traffic signal cycle to another while the MCF model only reproduces the repetition of a mean pattern. In counter part, mCF models are difficult to calibrate in order to fit macroscopic data. Moreover, several simulation runs are required with those models before obtaining representative results for a given traffic scenario. Indeed a large deviation in the results is expected due to large variability in driving behaviors.

\subsection{Noise source representations}

The emission law provides power noise levels $L w_{i}(t)$ of each vehicle $i$ on the network from its kinematics. All vehicles follow the same emission law. This hypothesis was validated in [23] for classical descriptors estimation in urban area, provided that traffic dynamics is precisely described. Nevertheless, it prevents the estimation of noise peaks ( $L_{\max }$ and $L_{1}$ ) that are due to noisy vehicles [23]. The law used in this study gives $L w$ with respect to speed and cruising mode (accelerating, cruising or decelerating) [14] ; see Figure 2.

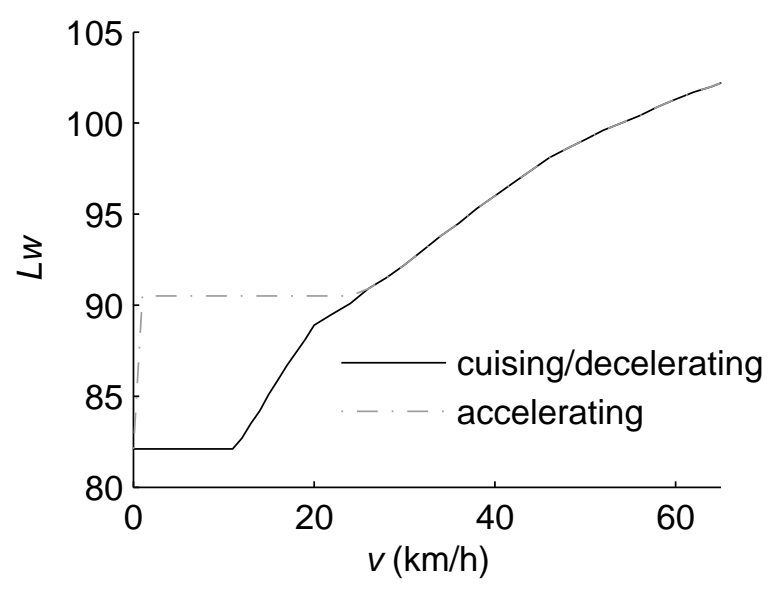

Figure 2: sound power emission law

\subsubsection{Vehicle line source representation}

The reference noise source representation is the vehicle line source (VLS). This is the finest one : each vehicle $i$ forms a segment source whose angle $\theta_{i, \Delta t}(t)$ seen from the receiver is defined by the positions between receiver and vehicle $i$ at $t$ and at $t+\Delta t$. This representation does not 
involve errors, but is useless in practice because it is time consuming: propagation calculation has to be determined at each time step.

\subsubsection{Fixed line sources representation}

Noise sources have to be gathered on line sources to reduce calculation times [13]. Power noise level $L W_{j}$ of a line source $j$ is deduced from the power noise level of vehicles on the line source (note that $L W$ refers to power noise level of a line source and $L w$ to power noise level of a vehicle):

$$
L W_{j}(t)=10 \log \left(\sum_{i \in J}{\frac{L_{0}}{l_{j}}}_{10}^{\frac{L w_{i}(t)}{10}}\right), J: \text { set of vehicles } i \text { inside the line source } j
$$

where $l_{\mathrm{j}}$ is the length of the line source, and $L_{0}=1 \mathrm{~m}$.

Then propagation calculation has only to be performed once at the beginning of the simulation: it gives the attenuation $\Delta_{\mathrm{j}, \mathrm{r}}$ between each line source $j$ and each receiver $r$. The result of this calculation is then applied at each time step to deduce the $L_{\text {Aeq,1s }}(t)$ at the receiver $r$. Only geometric sound propagation will be considered in this study to focus on noise dynamics due to traffic and noise source representations, and thus the $\Delta_{\mathrm{j}, \mathrm{r}}$ matrix is simplified. Note that sound propagation can also affect dynamics for shielded urban areas [24]. Within this hypothesis equivalent noise level $L_{\mathrm{Aeq}, 1 \mathrm{~s}}(t)$ is given by:

$$
L_{\text {Aeq, } 1 s}(t)=10 \log \left(\sum_{j} \alpha_{j} 1^{\frac{L W_{j}(t)}{10}}\right)-10 \log \left(2 \pi d / d_{0}\right)
$$

where $\alpha_{j}$ is the angle in radians of the line source $j$ seen from the receiver, $d$ is the distance between the road and the receiver, and $d_{0}=1 \mathrm{~m}$; see Figure 3 .

The cell length but also alignment between the cell and the receiver can affect noise estimation. Possible alignments range from "in front of" (receiver is in front of a cell) and "opposed" (receiver is between two cells, next to their joint boundary); see Figure 3.

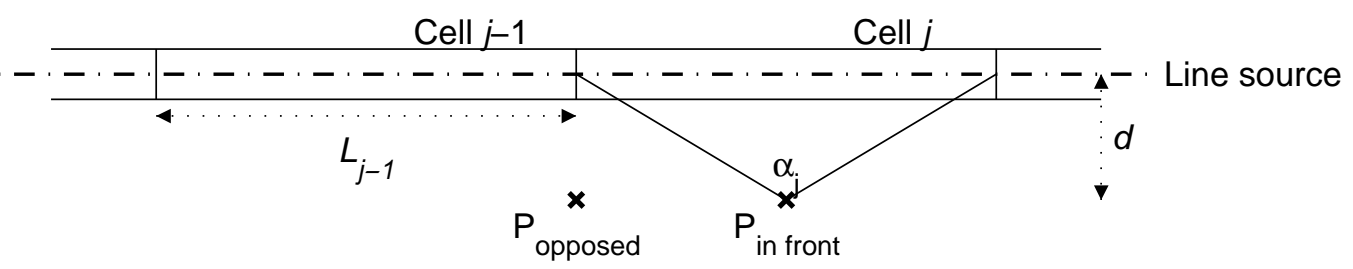

Figure 3: noise cells alignments

\subsection{Specific noise descriptors}


The use of specific descriptors is required to highlight traffic noise dynamics in the vicinity of signalized intersections. Indeed, the suitable scale to describe urban traffic noise is one traffic cycle and classical descriptors are not relevant at this scale [9]. Descriptors are calculated based on $L_{\text {Aeq,1s }}$ evolution. Descriptors used in this study are $L_{\text {Aeq, }}$ statistical descriptors $\left(L_{1}, L_{5}, L_{10}, L_{50}\right.$, $L_{90}$ ), $L_{\max }, L_{\min }$, but also specific descriptors that reveal noise dynamics at the traffic signal scale, based on [9]:

- The mean noise pattern. This is the pattern that repeats on average every traffic signal. The noise level corresponding to each instant $t_{\mathrm{i}}$ of a cycle $\left(0 \leq t_{\mathrm{i}}<t_{\text {cycle }}\right)$ is obtained by constructing the sample $S_{i}$ that contains the instants $t_{\mathrm{i}} \operatorname{modulo}\left(t_{\text {cycle }}\right)$, and then operating an acoustical average of the elements of $S_{i}$ whose level falls between $L_{90, \mathrm{Si}}$ and $L_{10, \mathrm{Si}}$ calculated from $S_{i \text {. }}$

- Specific descriptors that highlight characteristics of noise levels when traffic signal is green or red. Note that the following descriptors will be mentioned "specific descriptors" in the following of the paper. Specific descriptors are based on the $L_{\mathrm{Aeq}, 1 \mathrm{~s}}$ distribution and the mean noise pattern. An example is given in Figure 4, which looks like the patterns observed in situ in [9]. Note that these descriptors can only be calculated when the chronology of the traffic cycles is precisely known. An extraction procedure of these descriptors is proposed in [9] when the traffic cycle chronology is unknown. These descriptors are:

○ The green mode $m_{\text {green }}$ and the red mode $m_{\text {red }}$ of the Gaussian fit of the $L_{\text {Aeq,1s }}$ distribution, considering the levels received during the green phase and the red phase respectively; see Figure 4.

○ The A-weighted equivalent sound pressure levels $L_{\text {green }}$ and $L_{\text {red }}$ calculated when the traffic signal is green and red respectively.

○ The $L_{\text {green }}^{\prime}$ and $L_{\text {red, }}^{\prime}$ calculated from an acoustic average of the sample of $L_{\text {Aeq,1s }}$ between $L_{90}$ and $L_{10}$ during the green phase and the red phase respectively. $L^{\prime}$ green and $L_{\text {red }}^{\prime}$ correspond to the upper and lower levels of the mean noise pattern in front of a traffic signal, when it is constructed following the procedure above; see Figure 4. 

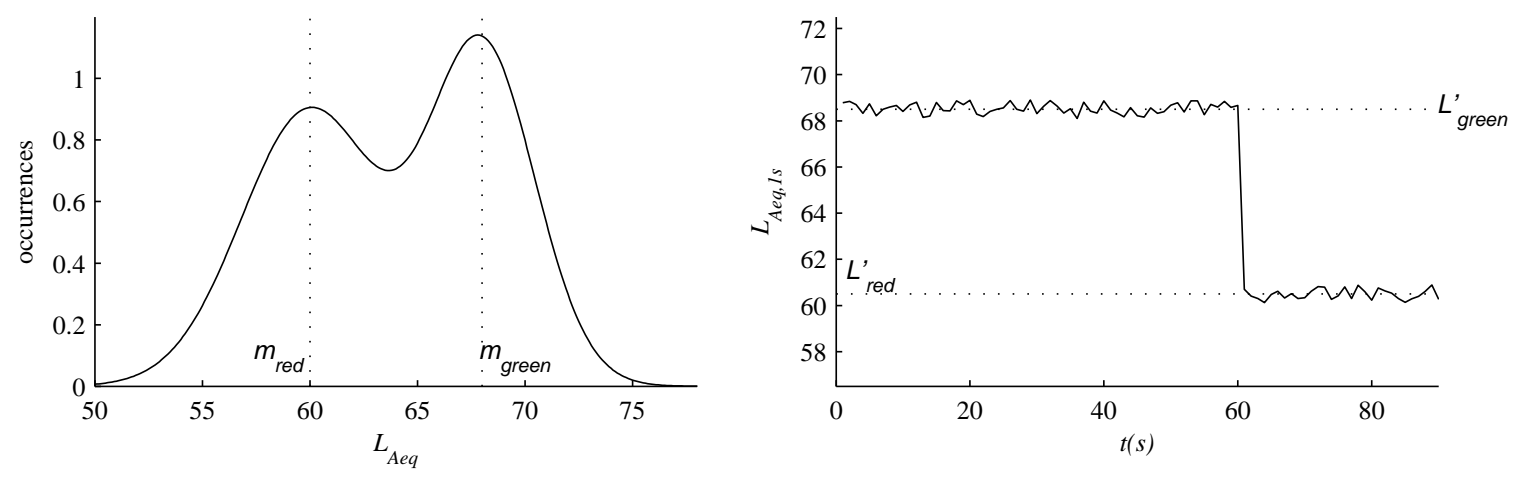

Figure 4: example of $L_{\mathrm{Aeq}, 1 \mathrm{~s}}$ distribution and mean noise pattern

\subsection{Methodology}

Noise source and traffic representations are tested separately to focus on their own influence: the influence of the noise source representation is tested in section 3 and the influence of the traffic representation is tested in section 4 . Tests are carried out on a $700 \mathrm{~m}$ one-lane road section, with a traffic signal TS located at $x_{\mathrm{TS}}=350 \mathrm{~m}$; see Figure 5. Durations of the green and red periods are $t_{\text {green }}=60 \mathrm{~s}$ and $t_{\text {red }}=30 \mathrm{~s}$. Traffic parameters are calibrated to match urban observations [25]: vehicle speed is limited to $V_{\mathrm{x}}=14 \mathrm{~m} / \mathrm{s}$, minimum spacing is $s_{\min }=5 \mathrm{~m}$, and wave speed is $w=-3.3 \mathrm{~m} / \mathrm{s}$. Finally, overtaking is forbidden.

The time step is fixed to $\Delta t=0.5 \mathrm{~s}$. Received levels $L_{\text {Aeq, } 1 \mathrm{~s}}(t)$ are calculated over a $2 \mathrm{~h}$ period. Receivers are located upstream, in front of, and downstream a traffic signal. Three distances from the road axis $(5.5 \mathrm{~m}, 10 \mathrm{~m}$ and $15 \mathrm{~m})$ are considered. Receivers are located at $2 \mathrm{~m}$ height.

\section{INFLUENCE OF NOISE SOURCE REPRESENTATION}

Influence of noise source representation is tested after fixing traffic representation to a MCF model, since this traffic representation is relevant to highlight noise dynamics [13]. Arriving flow rate is set to $Q_{1}=900 \mathrm{veh} / \mathrm{s}$, which corresponds to an unsaturated traffic condition upstream of the traffic signal (the queue formed during the red phase can vanish during the green phase). Vehicle line source (VLS) representation is used as a reference. Influence of cell length and alignment are jointly tested. Minimum cell length is fixed to $7 \mathrm{~m}$, which is the maximum distance that a vehicle can travel during a time step. Cell lengths have to be $2^{\text {nd }}$ power of $7 \mathrm{~m}$, in order to test all alignment configurations (see Figure 5). Hence the tested noise source representations are $7 \mathrm{~m}$ (LS7), 14m (LS14) and 28m (LS28) line sources. The following receiver positions are tested: $-28 m,-21 m,-14 m,-7 m, 0 m, 7 m, 14 m, 21 m$ and $28 m$ from the traffic signal. Both 
alignments "in front of" and "opposed" are covered for each line sources length with this map of receivers (see Figure 5).

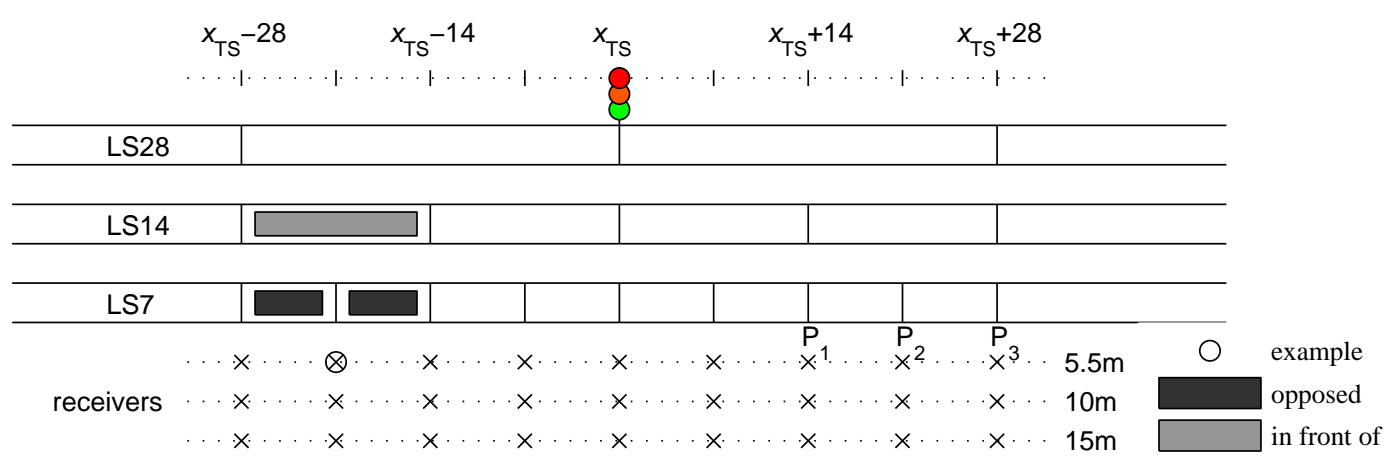

Figure 5: noise source representations and receivers positions

Impacts of line source length and alignment will be jointly evaluated, by considering the influence on descriptors estimation of doubling line source length whether alignment is changed or not. The accuracy of the mean noise pattern estimation will be assessed by calculating the standard deviation $\sigma$ between mean noise patterns obtained with (i) the considered noise source representation and (ii) the VLS representation.

LS7 vs LS14 and LS14 vs LS28 comparisons will be first discussed in details. One will focus on receivers located at $5.5 \mathrm{~m}$ from the road and downstream of the traffic signal, since noise dynamics is higher at this location. Cases investigated in details are:

- $\quad$ LS7 and LS14 comparison:

$\circ \quad \mathrm{P}_{1}$ (14m downstream); see Figure 5: LS7o (opposed) and LS14o (opposed) comparison. In this case cell length is doubled without changing alignment;

- $\mathrm{P}_{2}$ (21m downstream); see Figure 5: LS7o (opposed) and LS14f (in front) comparison. In this case cell length is doubled and alignment is changed.

- LS14 and LS28 comparison:

$\circ \quad P_{3}$ (28m downstream); see Figure 5: LS14o (opposed) and LS28o (opposed) comparison. In this case cell length is doubled without changing alignment;

- $\quad P_{1}$ (14m downstream); see Figure 5: LS14o (opposed) and LS28f (in front) comparison. In this case cell length is doubled and alignment is changed.

All the results will then be summarized in a final table to conclude on the suitable noise source representation with respect to the considered descriptor. 


\section{1. $\quad$ LS 7 and LS14 comparison}

\subsubsection{LS7o and LS14o comparison; $P_{1}\left(x=x_{T S}+14 m, d=5.5 m\right)$}

LS7o and LS14o representations are very similar. Energetic $\left(L_{\text {Aeq }}\right)$ and low levels descriptors $\left(L_{90}\right.$ and $\left.L_{50}\right)$ are estimated with errors under $1 \mathrm{~dB}(\mathrm{~A})$ with both representations; see Table1. Maximum levels estimation ( $L_{\max }, L_{1}$ and $L_{5}$ ) remains accurate with both LS7o and LS14o, with error under $2 \mathrm{~dB}(\mathrm{~A})$ (except for $L_{5}$ estimation with LS14o). Moreover, mean noise patterns are very similar with LS7o and LS14o to the VLS's one (see Figure 6a). Those patterns clearly highlight the distinction between high levels reached when traffic signal is green and low levels reached when traffic signal is red. Note that the periodic peaks observed when traffic signal is green (from $t=40 \mathrm{~s}$ to $t=60 \mathrm{~s}$ ) are due to the passing by of vehicles. Descriptors that represent upper and lower levels of the pattern $\left(L_{\text {green }}^{\prime}\right.$ and $\left.L_{\text {red }}^{\prime}\right)$ are also precisely estimated with both representations (error under $1 \mathrm{~dB}(\mathrm{~A})$ ).

\subsubsection{LS7o and LS14f comparison; $P_{2}\left(x=x_{T S}+21 \mathrm{~m}, d=5.5 \mathrm{~m}\right)$}

Both representations are relevant for noise dynamics assessment since (i) all descriptors are estimated with errors under $2 \mathrm{~dB}(\mathrm{~A})$, (ii) mean noise patterns are very close to the reference's one (see Figure 6b), (iii) specific descriptors are estimated with errors under $1 \mathrm{~dB}(\mathrm{~A})$; see Table1. Hence, doubling line sources length from $7 \mathrm{~m}$ to $14 \mathrm{~m}$ does not affect specific descriptors estimation and noise dynamics assessment, whatever the alignment between receiver and line source is.
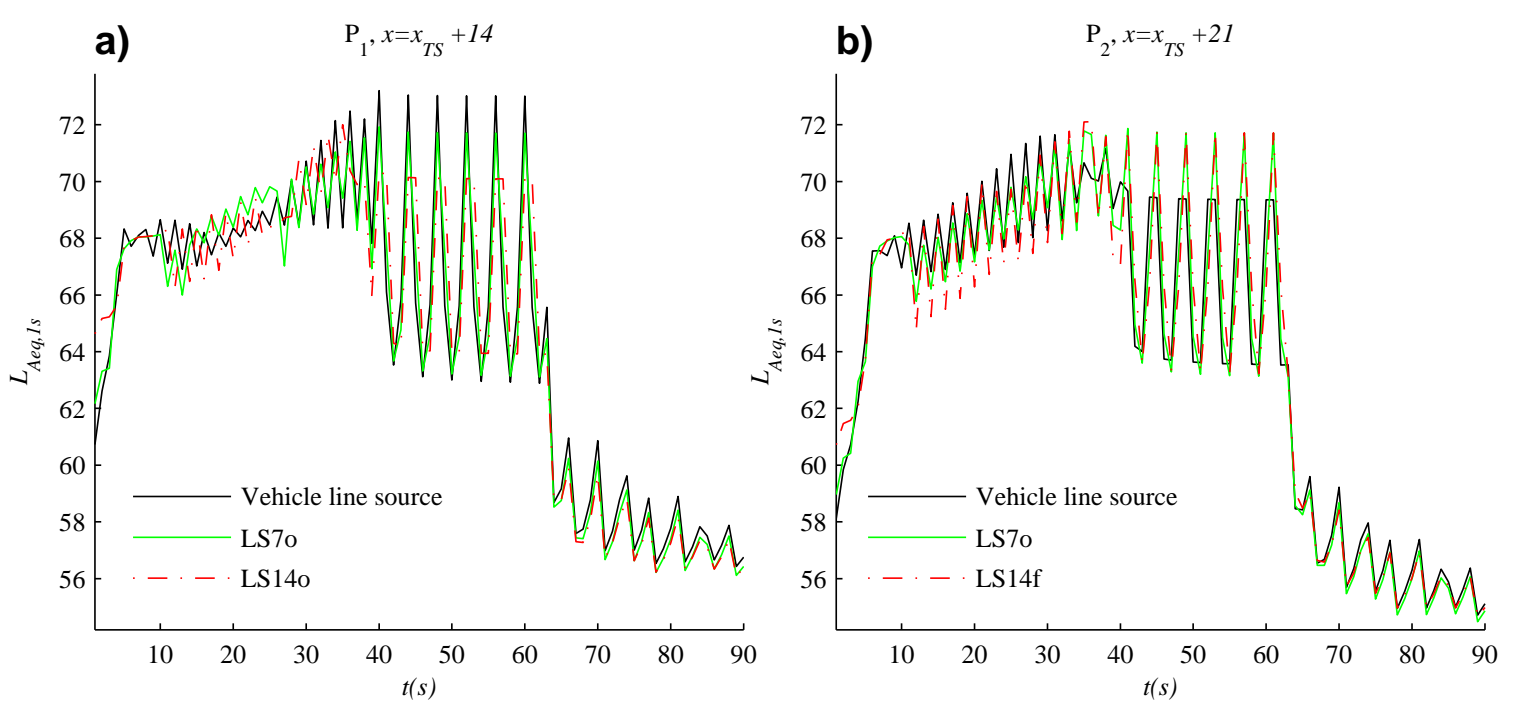

Figure 6: mean noise patterns at $\mathrm{P}_{1}$ and $\mathrm{P}_{2}$, for the following noise source representations: vehicle line source, $7 \mathrm{~m}$ line source and $14 \mathrm{~m}$ line source 


\begin{tabular}{|c|c|c|c|c|c|c|c|c|c|c|c|c|c|c|c|c|}
\hline $\begin{array}{l}\text { receiver } \\
\text { location }\end{array}$ & $\begin{array}{l}\text { noise source } \\
\text { representation }\end{array}$ & $L_{\text {Aeq }}$ & $L_{\max }$ & $L_{1}$ & $L_{5}$ & $L_{10}$ & $L_{50}$ & $L_{90}$ & $L_{\min }$ & $L_{\text {green }}$ & $L_{\text {red }}$ & $m_{\text {green }}$ & $m_{\text {red }}$ & $L_{\text {green }}^{\prime}$ & $L_{\text {red }}^{\prime}$ & $\sigma$ \\
\hline \multirow{4}{*}{$\begin{array}{c}\mathrm{P}_{1} \\
x=x_{\mathrm{TS}}+14\end{array}$} & VLS & 67.3 & 73.2 & 73.2 & 73.0 & 71.4 & 65.6 & 57.1 & 56.4 & 68.7 & 59.1 & 67.7 & 58.4 & 68.2 & 57.7 & - \\
\hline & LS7o & 67.0 & 71.9 & 71.9 & 71.7 & 70.6 & 66.3 & 56.8 & 56.1 & 68.5 & 58.9 & 67.7 & 58.0 & 67.7 & 57.5 & 0.9 \\
\hline & LS14o & 67.1 & 72.0 & 72.0 & 70.4 & 70.1 & 66.2 & 56.8 & 56.1 & 68.5 & 59.2 & 67.9 & 58.0 & 67.2 & 57.4 & 1.9 \\
\hline & LS28f & 67.2 & 71.5 & 71.2 & 70.4 & 69.5 & 68.0 & 59.8 & 59.2 & 68.5 & 61.3 & 68.3 & 60.5 & 68.4 & 59.8 & 2.5 \\
\hline \multirow{3}{*}{$\begin{array}{c}\mathrm{P}_{2} \\
x=x_{\mathrm{TS}}+21\end{array}$} & VLS & 66.9 & 71.6 & 71.6 & 71.0 & 70.0 & 64.6 & 55.6 & 54.7 & 68.3 & 58.4 & 67.4 & 57.1 & 67.8 & 56.4 & - \\
\hline & LS7o & 66.9 & 71.9 & 71.9 & 71.7 & 71.5 & 65.0 & 55.3 & 54.5 & 68.3 & 58.9 & 67.4 & 56.9 & 67.5 & 56.3 & 1 \\
\hline & LS14f & 66.9 & 72.1 & 72.1 & 71.7 & 71.6 & 66.2 & 55.5 & 54.7 & 68.3 & 59.1 & 67.4 & 57.0 & 67.4 & 56.3 & 1.4 \\
\hline \multirow{3}{*}{$\begin{array}{c}\mathrm{P}_{3} \\
x=x_{\mathrm{TS}}+28\end{array}$} & VLS & 67.4 & 73.2 & 73.2 & 73.0 & 71.6 & 65.6 & 54.6 & 53.5 & 68.8 & 59.1 & 67.4 & 56.3 & 68.1 & 55.2 & - \\
\hline & LS14o & 67.1 & 72.7 & 72.7 & 70.5 & 70.3 & 65.4 & 54.7 & 53.7 & 68.5 & 59.0 & 67.5 & 56.4 & 67.5 & 55.9 & 0.8 \\
\hline & LS280 & 67.1 & 72.9 & 72.8 & 70.6 & 70.2 & 65.5 & 55.6 & 54.8 & 68.5 & 59.3 & 67.5 & 57.1 & 67.3 & 56.3 & 1.9 \\
\hline
\end{tabular}

Table 1: Noise descriptors estimation at $\mathrm{P}_{1}, \mathrm{P}_{2}$ and $\mathrm{P}_{3}$, for vehicle line source (VLS), $7 \mathrm{~m}$ (LS7),

14m (LS14) and 28m (LS28) line source representations. In grey: error exceeding $1 \mathrm{~dB}(\mathrm{~A})$, in black: error exceeding $2 \mathrm{~dB}(\mathrm{~A})$

\subsection{LS14 and LS28 comparison}

\subsubsection{LS140 and LS280 comparison; $P_{3}\left(x=x_{T S}+28 \mathrm{~m}, d=5.5 \mathrm{~m}\right)$}

Energetic noise descriptors ( $L_{\mathrm{Aeq}}, L_{\mathrm{green}}$ and $L_{\mathrm{red}}$ ) are not affected by line source length; see Table1. Descriptors estimation with LS14o and LS28o are globally similar. Nevertheless:

- Differences in high levels estimation are observed with both LS14o and LS28o $\left(L_{5}\right.$ underestimation of more than $2 \mathrm{~dB}(\mathrm{~A})$ with both LS14o and LS280). It is due to the aggregation of energy on the line source, which affects the representation of vehicles passing in front of the receiver (see from $t=40$ s to $t=60$ s on Figure 7.a): dynamics linked to vehicle motion is partly lost.

- Estimation of low levels seems affected with LS28o (1.3 dB(A) overestimation of $L_{\min }$ ), since it hardly captures gaps without vehicles in front of the receiver. Finally, LS280 seems sufficient to assess specific descriptors estimation (see $L_{\text {green, }}^{\prime} L_{\text {red, }}^{\prime}$ $m_{\text {green }}$ and $m_{\text {red }}$ estimations; Table 1$)$. 

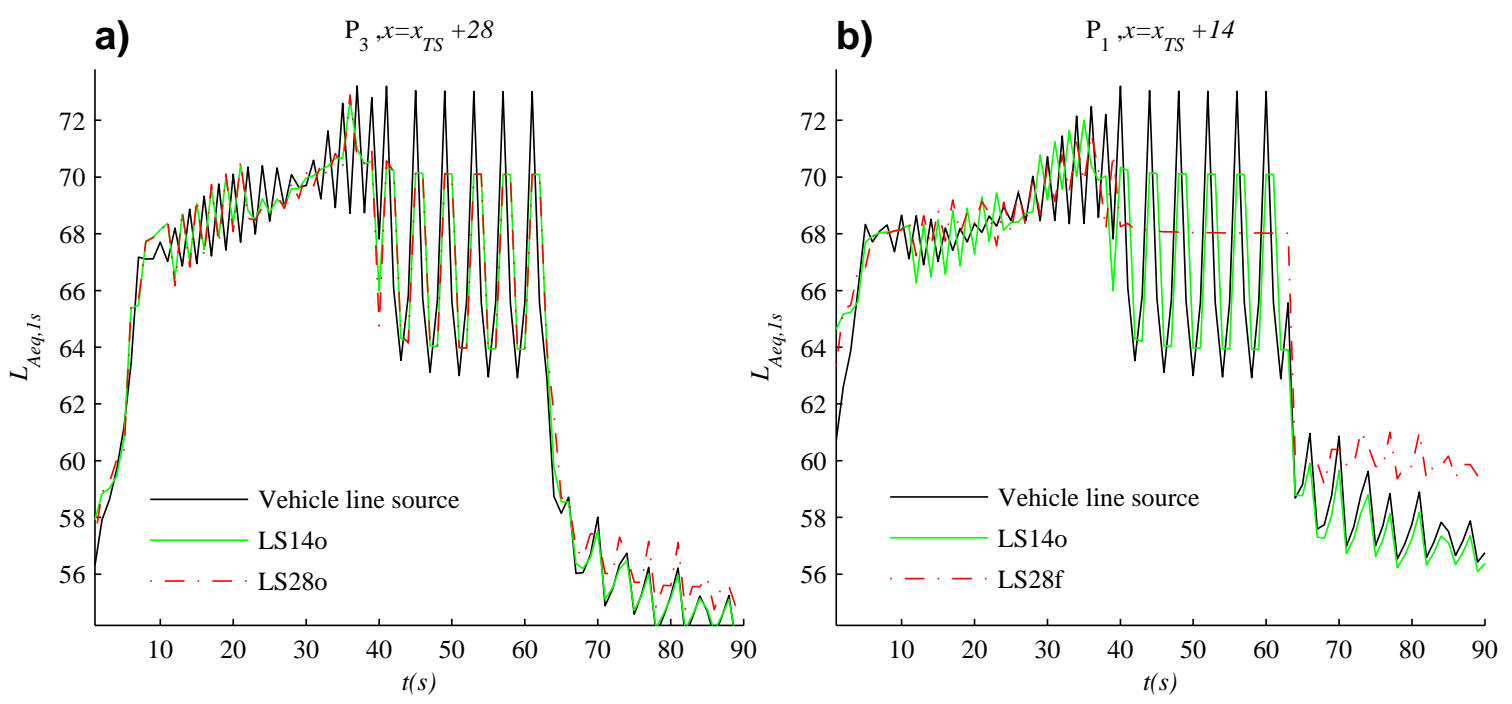

Figure 7: mean noise patterns at $\mathrm{P}_{3}$ and $\mathrm{P}_{1}$, for the following noise source representations: vehicle line source, $14 \mathrm{~m}$ line source, and $28 \mathrm{~m}$ line source

\subsubsection{LS14o and LS28f comparison; $P_{1}\left(x=x_{T S}+14 \mathrm{~m}, \mathrm{~d}=5.5 \mathrm{~m}\right)$}

Statistical descriptors and red levels estimation are affected by alignment with LS28f $(2.4 \mathrm{~dB}(\mathrm{~A})$ overestimation of $L_{50}$ and $2.2 \mathrm{~dB}(\mathrm{~A})$ overestimation of $L_{\text {red }}$ - see Table 1), while it remains precise with $\operatorname{LS140}\left(0.6 \mathrm{~dB}(\mathrm{~A})\right.$ overestimation of $L_{50}$ and $0.1 \mathrm{~dB}(\mathrm{~A})$ overestimation of $\left.L_{\mathrm{red}}\right)$. Mean noise pattern is quite far from the reference's one with LS28f $(\sigma=2.5 \mathrm{~dB}(\mathrm{~A}))$, because of low levels overestimation; see Figure 7b. It is linked to noise level estimation when traffic signal is red. This overestimation is due to vehicles that arrive at the back of the queue and are noisier than stopped vehicles; those vehicles enter sooner on the line source when it is larger. Moreover, noise dynamics from vehicles motion is totally lost with LS28f (see from $t=40 \mathrm{~s}$ to $t=60 \mathrm{~s}$ on Figure 7.b). However, $L_{\text {Aeq }}$ remains precisely estimated.

Thus alignment between line source and receiver can influence estimation with large line sources, especially sound levels when traffic signal is red. This could be troublesome since noise source representations have to be chosen to ensure an accurate estimation of noise dynamics wherever the receiver is located. The results for all receiver locations are now compared. Suitable line source lengths are discussed according to the descriptor to estimate.

\subsection{Suitable line source length}

Noise descriptors have been calculated for the $9 * 3=27$ points of Figure 5 . The noise source representations are compared at the location where the estimation is the worst. Maximum deviations from VLS representation among the 9 receivers of each distance from the road are 
shown for all descriptors in Table 2. It should not exceed a fixed value. Two bounds are considered: 1 and $2 \mathrm{~dB}(\mathrm{~A})$.

15m from the road: all descriptors (except $L_{\text {red }}^{\prime}$ ) can be estimated within a $1 \mathrm{~dB}(\mathrm{~A})$ error bound at any receiver location, even with LS28. Hence all the tested representations are suitable for noise dynamics assessment at $15 \mathrm{~m}$ from the road.

$10 \mathrm{~m}$ from the road: all descriptors can be estimated within a $2 \mathrm{~dB}(\mathrm{~A})$ error bound with LS28. LS28 is still relevant for energetic descriptors ( $L_{\mathrm{Aeq}}$ and $\left.L_{\text {green }}\right)$ estimation if only a $1 \mathrm{~dB}(\mathrm{~A})$ error bound is allowed, but it is not sufficient for statistical levels estimation. Note that LS14 is not sufficient too. LS7 guarantees estimation within $1 \mathrm{~dB}(\mathrm{~A})$ for all descriptors.

5.5m from the road: $L_{\text {Aeq }}$ can still be estimated within a $1 \mathrm{~dB}(\mathrm{~A})$ error bound with LS28. But this representation is not relevant for other descriptors. If a $2 \mathrm{~dB}(\mathrm{~A})$ error is allowed, LS14 is sufficient for descriptors relative to green and red phase estimation. This representation fails in estimating high levels ( $L_{\max }$ and $L_{1}$ ) accurately. Note that high level estimation is already impeded by noise emission laws, which do not distinguish the noisiest vehicles [23]. Finally, LS7 improves estimation and guarantees estimation of all descriptors within $2 \mathrm{~dB}(\mathrm{~A})$. It also guarantees estimation of all descriptors except high levels (from $L_{10}$ to $L_{\max }$ ) within a $1 \mathrm{~dB}(\mathrm{~A})$ error bound. 


\begin{tabular}{|c|c|c|c|c|c|c|c|c|c|c|c|c|c|c|c|}
\hline & & $L_{\text {Aeq }}$ & $L_{\max }$ & $L_{1}$ & $L_{5}$ & $L_{10}$ & $L_{50}$ & $L_{90}$ & $L_{\min }$ & $L_{\text {green }}$ & $L_{\text {red }}$ & $m_{\text {green }}$ & $m_{\text {red }}$ & $L_{\text {green }}^{\prime}$ & $L_{\text {red }}^{\prime}$ \\
\hline \multirow{3}{*}{$\begin{array}{l}15 \mathrm{~m} \\
\text { from the road }\end{array}$} & LS7 & -0.1 & -0.2 & -0.3 & 0.3 & 0.3 & -0.4 & -0.5 & -0.3 & -0.1 & -0.4 & -0.1 & -0.3 & 1.0 & -0.6 \\
\hline & LS14 & -0.2 & -0.4 & -0.7 & -0.6 & -0.5 & 0.5 & -0.5 & 0.2 & -0.1 & -0.6 & -0.1 & -0.5 & 1.0 & -0.9 \\
\hline & LS28 & -0.2 & 0.4 & -0.5 & -0.6 & -0.7 & -0.4 & 0.9 & -1.0 & -0.1 & -0.7 & 0.1 & -0.7 & -0.8 & -1.2 \\
\hline \multirow{3}{*}{$\begin{array}{l}10 \mathrm{~m} \\
\text { from the road }\end{array}$} & LS7 & -0.2 & 0.6 & 0.6 & 0.8 & 0.6 & 0.7 & -0.4 & -0.4 & -0.1 & -0.5 & -0.1 & -0.4 & -0.4 & -0.6 \\
\hline & LS14 & -0.3 & -1.4 & -1.4 & -1.4 & -1.3 & 1.0 & -0.6 & -0.4 & -0.2 & -0.9 & -0.1 & -0.7 & -0.6 & -1.1 \\
\hline & LS28 & -0.2 & 1.2 & -1.4 & -1.9 & -1.3 & 0.9 & 1.3 & 1.4 & -0.1 & -1.1 & 0.2 & -1.0 & -0.7 & -2.0 \\
\hline \multirow{3}{*}{$\begin{array}{c}5.5 \mathrm{~m} \\
\text { from the road }\end{array}$} & LS7 & -0.5 & 1.5 & 1.9 & 1.9 & 1.5 & 0.9 & -0.4 & -0.8 & -0.4 & -0.9 & -0.2 & -0.6 & 1.0 & 0.7 \\
\hline & LS14 & -0.6 & -2.8 & -2.9 & -2.9 & -2.9 & 1.7 & -0.9 & -1.4 & -0.4 & -1.5 & 0.2 & -1.1 & -0.9 & -1.0 \\
\hline & LS28 & -0.6 & -3.0 & -3.1 & -3.9 & -2.9 & 2.4 & 2.7 & 2.7 & -0.3 & 2.2 & 0.6 & 2.1 & 0.9 & 2.1 \\
\hline
\end{tabular}

Table 2: Maximum errors in noise descriptors estimation (compared to vehicle line source representation), for different line source lengths and distances from the road $(5.5 \mathrm{~m}, 10 \mathrm{~m}$ or $15 \mathrm{~m})$. in grey: error exceeding $1 \mathrm{~dB}(\mathrm{~A})$, in black: error exceeding $2 \mathrm{~dB}(\mathrm{~A})$

\section{INFLUENCE OF TRAFFIC REPRESENTATION}

Influence of traffic representation will be tested with a LS7 noise source representation, which is the most precise line source representation. The MCF traffic representation is used as a reference, since this traffic model is relevant for classical descriptors estimation [13]. The two following scenarios are considered to cover usual urban traffic conditions:

- Scenario 1: flow rate is $Q_{1}=900$ vehicle/h. This medium flow rate leads to the formation of a queue which vanishes over the cycle.

- Scenario 2: flow rate is $Q_{2}=1440$ vehicle/h. This high flow rate prevents the queue from discharging during the cycle. Thus the queue spills back on the network.

Alignment between line sources and receivers is kept constant (in position "opposed") in this section, to ensure comparison. Some receivers are located at higher distance from the traffic signal than in section 3, in order to cover all the deceleration and acceleration zones. Receiver points are located $-70 \mathrm{~m},-28 \mathrm{~m}, 0 \mathrm{~m}, 28 \mathrm{~m}$, and $70 \mathrm{~m}$ from the traffic signal. Mean noise patterns are derived for points located 5.5m from the road in Figure 8 and Figure 9.

\subsection{Influence of traffic resolution: MCL model vs MCF model}

MCL and MCF models are both based on a macroscopic behavior rule. The models only differ in their traffic representation: the former is macroscopic and the latter is individualized. Table3 
shows the maximum deviations in descriptors estimation between MCL and MCF model, among the 5 receiver locations.

If the flow rate is medium, MCL model is insufficient for all statistical descriptors estimation close to the road (3.5dB(A) deviation in $L_{50}$ estimation $5.5 \mathrm{~m}$ from the road; see Table 3$)$. On the contrary, $L_{\text {Aeq }}$ estimation is unbiased with both flow rates, even near the road axis.

\begin{tabular}{|c|c|c|c|c|c|c|c|c|c|c|c|c|c|c|c|}
\hline scenario & D & $L_{\text {Aeq }}$ & $L_{\max }$ & $L_{1}$ & $L_{5}$ & $L_{10}$ & $L_{50}$ & $L_{90}$ & $L_{\min }$ & $L_{\text {green }}$ & $L_{\text {red }}$ & $m_{\text {green }}$ & $m_{\text {red }}$ & $L_{\text {green }}^{\prime}$ & $L_{\text {red }}^{\prime}$ \\
\hline \multirow{3}{*}{1} & 15 & 0.1 & -1.0 & -1.0 & -0.9 & -0.9 & 0.8 & 2.2 & 3.2 & 0.2 & 0.3 & 0.3 & 0.8 & -0.5 & 1.2 \\
\hline & 10 & 0.2 & -1.5 & -1.5 & -1.6 & -1.9 & 1.7 & 2.8 & 4.6 & 0.2 & 0.4 & 1.1 & 2.0 & -1.1 & 1.5 \\
\hline & 5.5 & 0.1 & -2.2 & -2.1 & -2.6 & -3.2 & 3.5 & 5.0 & 6.8 & 0.3 & -0.5 & 2.7 & 3.7 & 2.6 & 2.4 \\
\hline \multirow{3}{*}{2} & 15 & -0.1 & -0.4 & -0.4 & -0.3 & -0.2 & 0.1 & -0.4 & -0.3 & -0.1 & -0.7 & 0.2 & -0.5 & -1.0 & -0.7 \\
\hline & 10 & -0.1 & -0.7 & -0.7 & -0.5 & -0.4 & 0.3 & -0.5 & -0.5 & -0.1 & -0.9 & 0.5 & -0.6 & -2.3 & -1.5 \\
\hline & 5.5 & -0.1 & -1.3 & -1.3 & -1.2 & -1.1 & 1.4 & -0.8 & -0.7 & 0.2 & -1.4 & 1.7 & 1.8 & -0.4 & -0.8 \\
\hline
\end{tabular}

Table 3: Maximum deviations among the five receivers $(-70 m,-28 m, 0 m, 28 m$, and $70 m$ from TS) between MCF and MCL models, for scenarios 1 and 2 and different distances from the road $(5.5 \mathrm{~m}, 10 \mathrm{~m}$ and $15 \mathrm{~m})$. in grey: error exceeding $1 \mathrm{~dB}(\mathrm{~A})$, in black: error exceeding $2 \mathrm{~dB}(\mathrm{~A})$ 

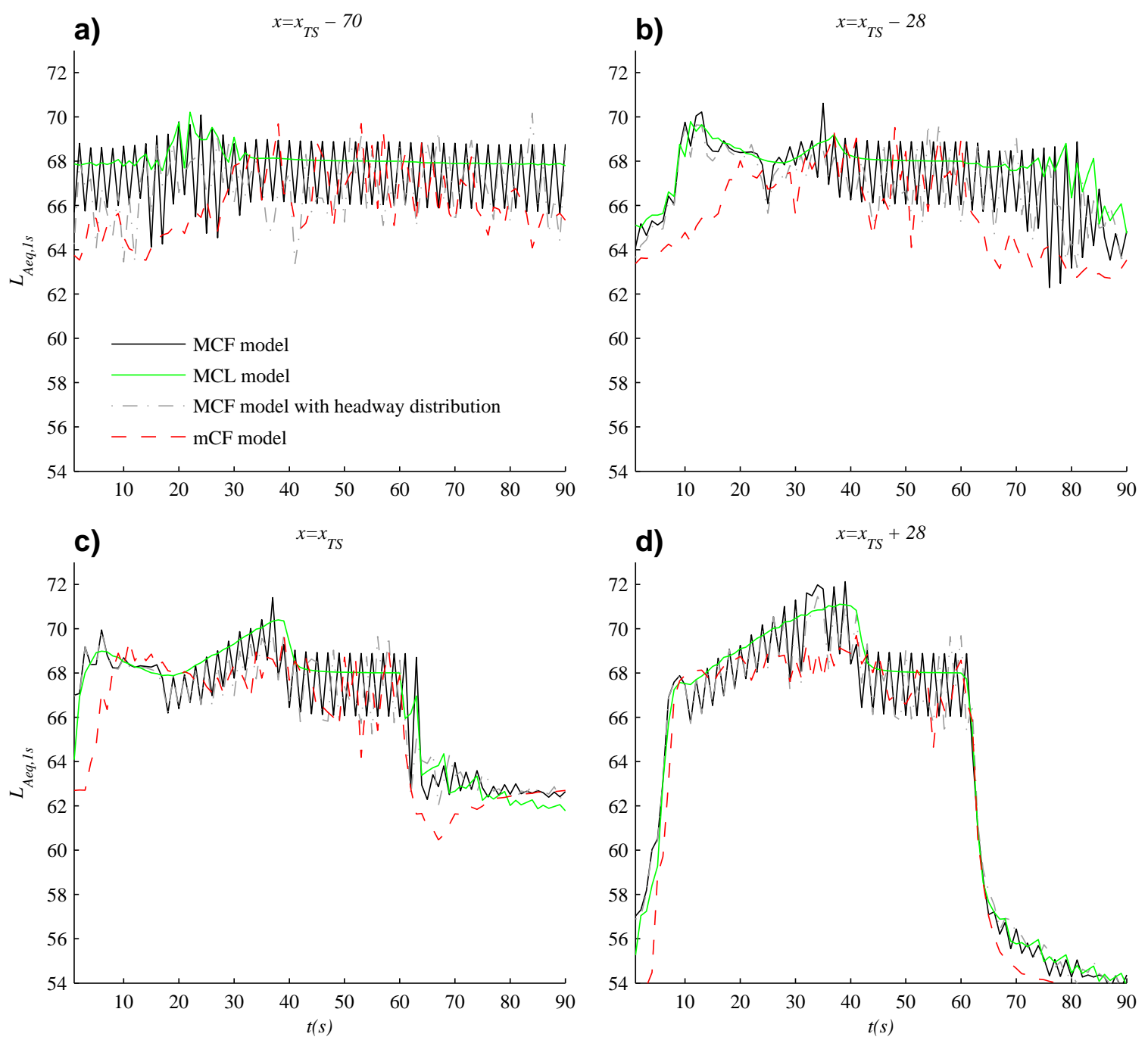

Figure 8: mean noise patterns at a) $x=x_{T S}-70$, b) $x=x_{T S}-28$, c) $x=x_{T S}$ and d) $x=x_{T S}+28,5.5 \mathrm{~m}$ from the road, for the following traffic representations: MCF model, MCL model, MCF model with headway distributions, and mCF model. $Q=900 \mathrm{veh} / \mathrm{h}$

Moreover, Figure 8 and Figure 9 show that mean noise patterns with MCL model are similar to MCF's ones, whatever the flow rate and the receiver location are. Thus $L_{\text {green }}$ and $L_{\text {red }}$ estimations are unbiased since traffic dynamics close to the TS is precisely described. Finally, MCL representation cannot guarantee specific descriptors estimation close to the road axis: Table 3 shows $2.7 \mathrm{~dB}(\mathrm{~A})$ overestimation of $m_{\text {green }}$ and $3.7 \mathrm{~dB}(\mathrm{~A})$ overestimation of $m_{\text {red }}$ at some location. Note that those errors occur upstream of the TS. They are due to the macroscopic resolution that averages vehicle kinematics, whereas distinction in behaviors is kept with the MCF model that considers the proper emission of each vehicle and adds them afterwards. 
a)

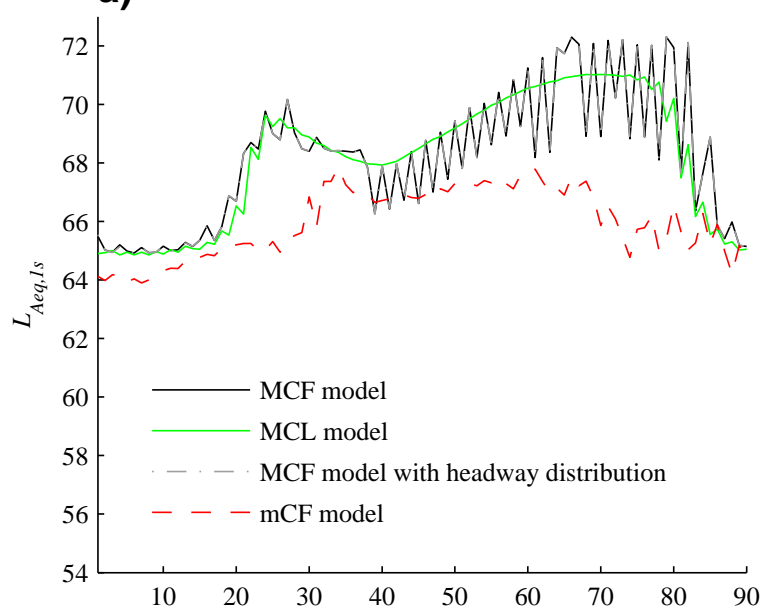

c)

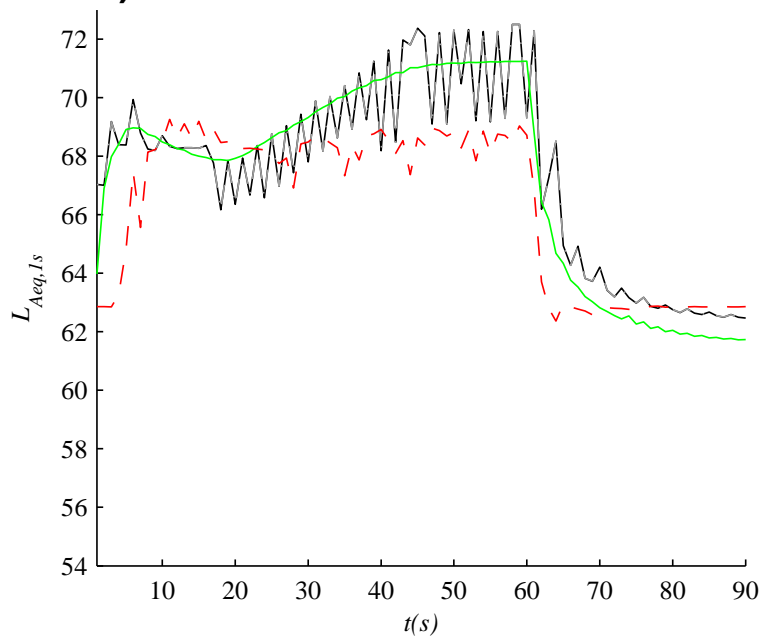

b)

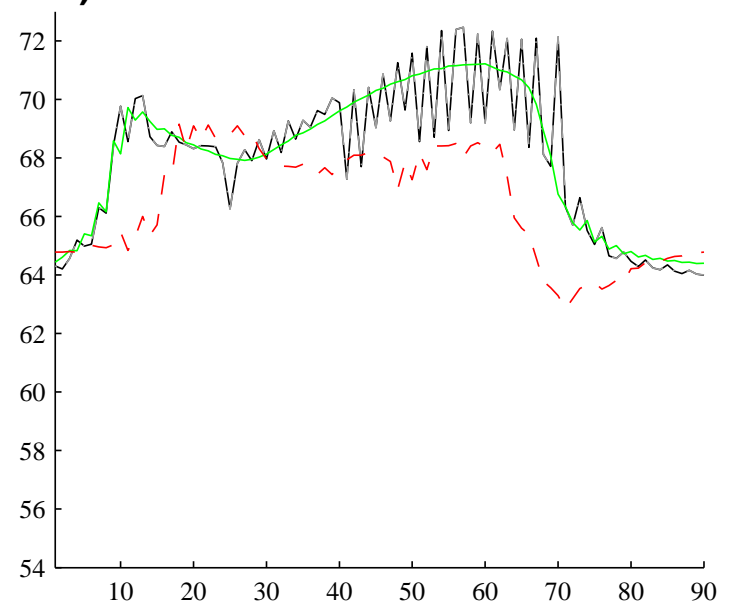

d)

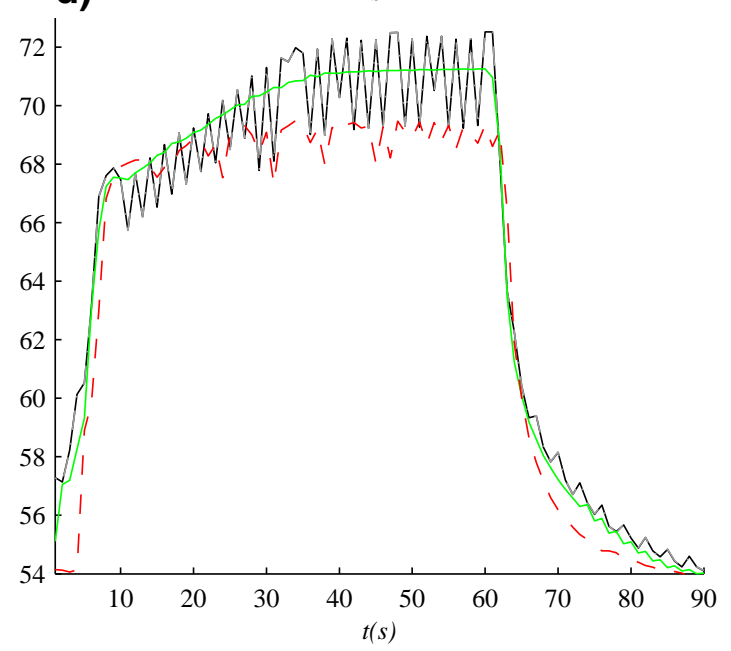

Figure 9: mean noise patterns at a) $x=x_{T S}-70$, b) $x=x_{\mathrm{TS}}-28$, c) $x=x_{\mathrm{TS}}$ and d) $x=x_{\mathrm{TS}}+28,5.5 \mathrm{~m}$ from the road, for the following traffic representations: MCF model, MCL model, MCF model with headway distributions, and mCF model. $Q=1440 \mathrm{veh} / \mathrm{h}$

\subsection{Influence of headway distributions}

Headway distributions can be used in MCF model to represent stochastic arrivals of vehicles. Can et al. have shown that those distributions affect low sound levels since they allow for catching large gaps between vehicles [13]. This is confirmed by Table4 which shows the maximum deviations in descriptors estimation due to headway distributions among the 5 receiver locations.

Headway distributions have no influence on mean noise patterns, since they affect low levels that are filtered by the mean noise pattern reconstitution process; see Figure 8 and Figure 9 . Headway distributions also have a limited impact on specific noise descriptors. When flow rate is high, deviations fall under $2 \mathrm{~dB}(\mathrm{~A})$ for each specific descriptor whatever the location is. It is 
due to the queue that climbs back the network and set its dynamics even if vehicle arrivals are distributed. When flow rate is medium, deviations that exceed $1 \mathrm{~dB}(\mathrm{~A})$ are observed in $L^{\prime}$ green and $L_{\text {red }}^{\prime}$ estimates. They correspond to errors at the receiver located $70 \mathrm{~m}$ upstream, where the queue does not climb back. Those deviations are due to the arrivals of vehicles that are not influenced by the queue.

\begin{tabular}{|c|c|c|c|c|c|c|c|c|c|c|c|c|c|c|c|}
\hline scenario & D & $L_{\text {Aeq }}$ & $L_{\max }$ & $L_{1}$ & $L_{5}$ & $L_{10}$ & $L_{50}$ & $L_{90}$ & $L_{\min }$ & $L_{\text {green }}$ & $L_{\text {red }}$ & $m_{\text {green }}$ & $m_{\text {red }}$ & $L_{\text {green }}^{\prime}$ & $L_{\text {red }}^{\prime}$ \\
\hline \multirow{3}{*}{1} & 15 & -0.5 & 1.2 & 0.7 & 0.8 & 0.7 & 0.5 & -3.1 & -10.3 & 0.2 & 0.3 & 0.5 & 0.6 & 1.2 & 0.5 \\
\hline & 10 & -0.6 & 0.9 & 0.5 & 0.5 & 0.5 & 1.1 & -3.4 & -10.7 & 0.2 & 0.3 & 1.1 & 0.8 & 0.6 & 1.2 \\
\hline & 5.5 & -0.6 & 0.8 & 0.3 & 0.2 & 0.4 & 2.1 & -3.5 & -10.9 & 0.2 & 0.3 & 2.0 & 1.2 & 1.0 & 0.5 \\
\hline \multirow{3}{*}{2} & 15 & -0.1 & 0.0 & 0.0 & 0.0 & 0.0 & 0.1 & 0.1 & -1.2 & 0.0 & 0.0 & 0.0 & 0.0 & 0.1 & 0.0 \\
\hline & 10 & -0.1 & 0.0 & 0.0 & 0.0 & 0.0 & 0.0 & 0.1 & -1.7 & 0.0 & 0.0 & 0.0 & 0.0 & 0.0 & -0.4 \\
\hline & 5.5 & 0.0 & 0.0 & 0.0 & 0.0 & 0.1 & 0.0 & 0.1 & -3.2 & 0.0 & 0.0 & 0.0 & 0.0 & 0.0 & 0.0 \\
\hline
\end{tabular}

Table 4: Deviations between MCF model with and without headway distributions, for scenarios 1 and 2 and different distances from the road $(5.5 \mathrm{~m}, 10 \mathrm{~m}$ and $15 \mathrm{~m})$. Each value corresponds to the maximum deviations among the five receivers $(-70 m,-28 m, 0 m, 28 m$, and $70 m$ from TS). in grey: error exceeding $1 \mathrm{~dB}(\mathrm{~A})$, in black: error exceeding $2 \mathrm{~dB}(\mathrm{~A})$

\subsection{Influence of traffic representation: MCF model vs mCF model}

Dynamics set by the traffic cycle is highlighted by both models (see Figure 8 and Figure 9), but differences in behavior rules involve local differences in descriptors estimation; see Table 5. Noise estimation is quite similar for both models in front of the TS (see noise patterns in Figure 8.c and Figure 9.c). Most of the large deviations observed in Table 5 for scenario 2 occur at the two points located $28 \mathrm{~m}$ and $70 \mathrm{~m}$ upstream of the TS. This depicts the differences in the queue propagation modeling by both models. Moreover, the difference in noise descriptors estimation shows the difficulty in calibrating mCF models. In fact, vehicles characteristics are individually distributed in the mCF model while they must agree with macroscopic data. For example, to obtain a mean speed of $14 \mathrm{~m} / \mathrm{s}$, one can set in $\mathrm{mCF}$ a distribution centered on $15.5 \mathrm{~m} / \mathrm{s}$ with a standard deviation of $1.5 \mathrm{~m} / \mathrm{s}$. In fact when performing replications vehicle speeds are set by the slowest one. Hence stochasticity in vehicle speeds can affect the average flow speed and then the noise emitted.

Individualization of behaviors in $\mathrm{mCF}$ models also influences the mean noise pattern downstream and in front of the traffic signal: green levels are constant in time with the mCF model while they increase with the MCF model; see from $t=10$ s to $t=60$ s in Figure 8.c,d and 
Figure 9.c,d. This is due to dispersion in behaviors from one cycle to another with the mCF model. Indeed, speed of each platoon of vehicles is fixed by its slowest vehicle (overtaking is forbidden in this study), and can vary from one cycle to another. This gives constant levels when averaging over cycles. Note that [9] has shown that for a one way three lanes road, green levels are constant in front of the traffic signal and increasing downstream.

\begin{tabular}{|c|c|ccccccccccccccc|}
\hline scenario & $\mathrm{D}$ & $L_{\text {Aeq }}$ & $L_{\max }$ & $L_{1}$ & $L_{5}$ & $L_{10}$ & $L_{50}$ & $L_{90}$ & $L_{\min }$ & $L_{\text {green }}$ & $L_{\text {red }}$ & $m_{\text {green }}$ & $m_{\text {red }}$ & $L_{\text {green }}^{\prime}$ & $L_{\text {red }}^{\prime}$ \\
\hline \multirow{3}{*}{1} & 15 & -1.5 & 2.0 & 1.0 & -1.0 & -1.2 & -2.4 & -3.6 & -10.2 & -1.0 & -1.9 & -1.5 & -2.3 & -1.4 & -1.7 \\
& 10 & -1.6 & 2.5 & 1.0 & -1.1 & -1.3 & -2.7 & -4.0 & -10.5 & -1.1 & -2.2 & -1.8 & -1.7 & -1.9 & -1.4 \\
& 5.5 & -1.8 & 2.9 & 1.1 & -1.2 & -2.1 & -2.7 & -4.1 & -10.7 & -1.1 & -2.5 & -2.2 & -0.8 & -1.5 & -1.5 \\
& 15 & -2.6 & -2.0 & -2.6 & -3.0 & -2.9 & -2.2 & -1.3 & -2.1 & -1.7 & -3.4 & -2.0 & -4.0 & -3.0 & -2.8 \\
2 & 10 & -2.7 & -2.2 & -2.9 & -3.4 & -3.3 & -2.2 & -1.2 & -2.7 & -1.7 & -3.6 & -2.2 & -3.6 & -2.0 & -3.1 \\
& 5.5 & -2.9 & -2.3 & -3.0 & -3.9 & -4.1 & -2.5 & -1.2 & -4.6 & -1.7 & -3.7 & -2.5 & -2.5 & -2.5 & -3.7 \\
\hline
\end{tabular}

Table 5: Deviations between MCF and mCF models, for scenarios 1and 2 and different distances from the road (5.5m, $10 \mathrm{~m}$ and $15 \mathrm{~m})$. Each value corresponds to the maximum deviations among the five receivers $(-70 \mathrm{~m},-28 \mathrm{~m}, 0 \mathrm{~m}, 28 \mathrm{~m}$, and $70 \mathrm{~m}$ from $\mathrm{TS})$ in grey: error exceeding $1 \mathrm{~dB}(\mathrm{~A})$, in black: error exceeding $2 \mathrm{~dB}(\mathrm{~A})$

Finally, noise levels are higher in the beginning of the red phase and upstream to the TS with MCF model; see from $t=60 \mathrm{~s}$ to $t=70 \mathrm{~s}$ on Figure $8 \mathrm{~b}$. and Figure $9 \mathrm{~b}$. Indeed, vehicles are decelerating when approaching the TS and are then less noisy; this deceleration is not represented in MCF models. Note that this can be easily corrected in practice in MCF models by fixing a posteriori the speed and acceleration of vehicles that are approaching.

\section{DISCUSSION}

The goal of this paper was to determine suitable noise source and traffic representations to capture traffic noise dynamics in urban area. Representations have been separately tested, with scenarios that reflect usual traffic situations in the nearby of traffic signals. Receivers were located upstream, in front of, and downstream a traffic signal, at three distances from the road: $5.5 \mathrm{~m}, 10 \mathrm{~m}$ and $15 \mathrm{~m}$. Comparisons were based on specific descriptors estimations over a $2 \mathrm{~h}$ period. Those descriptors are sensible to noise dynamics at the traffic scale: they highlight the noise pattern that repeats on average every traffic signal, and focus on the noise levels reached during the green and the red phase [9]. 
Concerning noise representations, alignment between line source and receivers can affect estimation with large line sources $(28 \mathrm{~m})$. Thus line source length has to be carefully set to guarantee accurate descriptors estimation on a grid of receivers. $28 \mathrm{~m}$ line source length seems sufficient for noise descriptors estimation beyond $10 \mathrm{~m}$ from the road if a $2 \mathrm{~dB}(\mathrm{~A})$ error is allowed. The line source length must be reduced when receivers are closer to the road $(5.5 \mathrm{~m})$. However, $L_{\text {Aeq }}$ remains precisely estimated (error below $1 \mathrm{~dB}(\mathrm{~A})$ ) close to the road with $28 \mathrm{~m}$ line sources provided that traffic dynamics is precisely described. $14 \mathrm{~m}$ line source length is sufficient to assess specific descriptors ( $\left.L_{\text {green }}^{\prime} L_{\text {red }}^{\prime}, m_{\text {green }}, m_{\text {red }}\right)$ at $5.5 \mathrm{~m}$ from the road within a $2 \mathrm{~dB}(\mathrm{~A})$ error bound. LS7 ensures their estimation within a $1 \mathrm{~dB}(\mathrm{~A})$ error. Finally, peaks of noise $\left(L_{1}\right.$ and $L_{\max }$ ) cannot be precisely estimated through line source representation. This conclusion might have been different if specific noise laws that distinguish the noisiest vehicles from the others had been used as explained in [23].

Concerning traffic representations, the macroscopic conservation law model is sufficient to estimate correctly $L_{\text {Aeq }}$ whatever the distance from the road is. Nevertheless, it fails in capturing dynamics triggered by the traffic signal, if distances from the road axis are below $15 \mathrm{~m}$. Carfollowing models that explicitly represent vehicle trajectories are then necessary to highlight this dynamics.

Taking headway distributions into account makes it possible to represent dispersion in arrivals of vehicles. It does not improve specific descriptors estimation, since those descriptors highlight noise dynamics at the traffic signal scale, which is mainly set by the traffic signal. Yet, distributions can improve low levels estimation since they allow for representing gaps between vehicles.

Finally, use of a microscopic or a macroscopic behavior rule for descriptors estimation can be discussed. Models are difficult to compare since it is difficult to precisely calibrate mCF models so as to fit the observed macroscopic traffic data (flow rates, mean speeds, etc.), which can be a drawback of mCF models in practice for urban traffic noise prediction. However, with the hypothesis chosen for this study, the mCF model seems more accurate to assess noise dynamics in front of a traffic signal. On the contrary, downstream of the traffic signal, the MCF model better represents the shape of the pattern observed in [9] from measurements. Finally, both models seem able to capture the dynamics set by the traffic signal. Recent work has shown that MCF models were easy to calibrate on a real case study, and fit experimental data coupled with $14 \mathrm{~m}$ line sources representation [26]. The ability of $\mathrm{mCF}$ models to reproduce real urban noise levels has now to be tested.

\section{ACKNOWLEDGEMENTS}


The authors would like to thank Estelle Chevalier, for her careful reading that greatly improved the quality of the paper. This research was partly founded by "the Région Rhône-Alpes".

\section{REFERENCES}

[1] Steele, C., A critical review of some traffic noise prediction models. Applied Acoustics, 2001. 62: p. 271-287.

[2] Leclercq, L., A traffic flow model for dynamic estimation of noise. 2002: Phd report. 317 p. (in french, see (Giorgi and al., 2002) for information in english).

[3] Leclercq, L. and Lelong J. Dynamic evaluation of urban traffic noise. in Adriano Alippi, Proceedings of the 17th International congress on Acoustics. 2001. Rome. 2 p.

[4] Leclercq, L., Lelong J. and Defrance J. Dynamic assessment of road traffic noise: elaboration of a global model. in 18th congress on acoustics. 2004. Kyoto. 6 p.

[5] De Coensel, B., De muer T., Yperman I. and Botteldoren D., The influence of traffic flow dynamics on urban soundscape. Applied Acoustics, 2005. 66: p. 175-194.

[6] Rotranomo, Final conference on 28.09.2005 [on line]. 2005, http://www.rotranomo.com/.

[7] Oshino, Y., Tsukui K., Hanabusa H., Bhaskar A., Chung E. and Kuwahara M. Study on road traffic noise prediction model taking into account the citywide road network. in Inter-noise. 2007. Istanbul. $8 \mathrm{p}$.

[8] De Coensel, B., Botteldoren, D., Vanhove, F. \& Logghen, S. Microsimulation Based Corrections on the Road Traffic Noise Emission near Intersections. Acta acustica united with acustica, 2007. 93: p.24125.

[9] Can, A., Leclercq, L., Lelong, J. et Defrance, J. Capturing urban traffic noise dynamics through relevant descriptors. Applied Acoustics (2007), doi:10.1016/j.apacoust.2007.09.006.

[10] Can, A., Leclercq, L. and Lelong, J. Acoustic descriptors for dynamic noise estimation close to traffic signals. in The 36th International Congress and Exhibition on Noise Control Engineering (InterNoise). 2007. Istanbul, Turkey. 10p.

[11] Forssén, J., Hornikx, M., Statistics of A-Weighted Road Traffic Noise in Shielded Urban Areas. Acta Acustica United with Acustica, 2006. 92: p. 998-1008.

[12] Genuit, K., Fiebig, A. Psychoacoustics and its benefit for the soundscape approach. Acta Acustica united with Acustica.2006. 92: p. 952-958.

[13] Can, A., Leclercq, L. et Lelong, J. Dynamic estimation of urban traffic noise: influence of traffic and noise source representations. Applied Acoustics (2007), doi:10.1016/j.apacoust.2007.05.014.

[14] Besnard, F., et al. The procedure for updating the vehicle noise emissions values of the French "Guide du Bruit des transports terrestres". in EAA European Acoustics Association Euronoise. 2003. Naples.

[15] May, A., Traffic flow fundamentals. 1990: Prentice-Hall, Englewood Cliffs, New Jersey. 464 p.

[16] Newell, G.F., A simplified theory of kinematic waves in highway traffic, Part I : general theory. Transportation Research Part B, 1993. 27B(4): p. 281-287 
[17] Daganzo, C.F., A variational formulation of kinematic waves : basic theory and complex boudary conditions. Transportation Research Part B, 2005. 39: p. 187-196

[18] Newell, G., A simplified car-following theory: a lower order model. Transportation Research Part B, 2002. 36: p. 195-205.

[19] Leclercq, L., Laval J. and Chevallier E., The Lagrangian coordinates and what does it means for first order traffic flow models, Proceedings of the 17th International Symposium on Transportation and Traffic Theory. Accepted for publication.

[20] Gazis, D.C., Herman R. and Potts R.B., Car following theory of steady traffic. Operation Research, 1959. 7: p. 499-505.

[21] Chandler, R.E., Herman R. and Montroll E.W., Traffic dynamics : studies in car following. Operations research, 1958. 6: p. 165-184.

[22] Barcelo, J. and Casas J. Dynamic network simulation with AIMSUN. in International Symposium on Transport Simulation. 2002. Yokohama: Ed. Kluwer. 25 p.

[23] Can, A., Leclercq, L. and Lelong, J. Dynamic urban traffic noise: do individualized emission laws improve estimation? in 19th International Congress on Acoustics (ICA). 2007. Madrid, Spain. 6 p.

[24] Forssén, J. and Hornikx M. Statistics of road traffic noise levels in shielded urban areas. in internoise. 2007. Istanbul. 8 p.

[25] Leclercq,L. Calibration of Flow-Density Relationships on Urban Streets Transportation Research Record: Journal of the Transportation Research Board, 2005. 1934: p.226-234

[26] Leclercq, L., et al., Dynamic noise estimation in urban area: case study (in French: Estimation dynamique du bruit de circulation en milieu urbain : étude d'un cas réel) R.I.L. $\mathrm{N}^{\circ} 0703$, Editor. 2007: p. 65. 